\title{
Treatment of polypoidal choroidal vasculopathy by intravitreal injection of bevacizumab.
}

\section{$\operatorname{AUTHOR(S):~}$}

Tsujikawa, Akitaka; Ooto, Sotaro; Yamashiro, Kenji; Tamura, Hiroshi; Otani, Atsushi; Yoshimura, Nagahisa

\section{CITATION:}

Tsujikawa, Akitaka ... [et al]. Treatment of polypoidal choroidal vasculopathy by intravitreal injection of bevacizumab. Japanese journal of ophthalmology 2010, 54(4): 310-319

\section{ISSUE DATE:}

2010-07

URL:

http://hdl.handle.net/2433/130712

\section{RIGHT:}

The original publication is available at www.springerlink.com; この論文 は出版社版でありません。引用の際には出版社版をご確認ご利用くだ さい。; This is not the published version. Please cite only the published version. 


\section{Title page}

Title: Treatment of Polypoidal Choroidal Vasculopathy with Intravitreal Injection of

Bevacizumab

Authors: Akitaka Tsujikawa, MD, Sotaro Ooto, MD, Kenji Yamashiro, MD, Hiroshi

5 Tamura, MD, Atsushi Otani, MD, and Nagahisa Yoshimura, MD

Institutions: Department of Ophthalmology and Visual Sciences, Kyoto University

Graduate School of Medicine, Kyoto, Japan

Running title: Bevacizumab for PCV

Correspondence to: Akitaka Tsujikawa, Department of Ophthalmology and Visual

Sciences, Kyoto University Graduate School of Medicine, 54 Shogoin-Kawahara-cho,

Sakyo-ku, Kyoto 606-8507, Japan.

fax: +81-75-752-0933; tel: +81-75-751-4875; e-mail: tujikawa@kuhp.kyoto-u.ac.jp

The authors have no financial interest in the materials or devices mentioned in this

15 article. 


\begin{abstract}
Purpose: To evaluate the efficacy of intravitreal bevacizumab (IVB) in eyes with polypoidal choroidal vasculopathy (PCV).
\end{abstract}

Methods: Seventeen eyes of 16 patients with subfoveal or juxtafoveal PCV were

5 treated with IVB. As an initial treatment, $\underline{10}$ eyes were treated with a single injection of bevacizumab and $\underline{7}$ eyes were treated with 3 monthly injections. Additional IVB injections were performed in $\underline{15}$ eyes when a recurrent or residual exudative change was seen. Follow-up after initiation of IVB ranged from 12 to 30 months (mean, $\underline{20.7}$ \pm 5.7 months).

Results: The mean foveal thickness before IVB $(\underline{492 \pm 205} \mu \mathrm{m})$ was decreased to $\underline{384 \pm 181} \mu \mathrm{m}$ at three months $(P=\underline{0.0008})$, and with additional IVB for recurrent exudative changes, the foveal thickness was still reduced significantly at 12 months $(\underline{392 \pm 203} \mu \mathrm{m}, P=\underline{0.0270}) . \quad$ Mean visual acuity at baseline $(\underline{0.54 \pm 0.38}$ in logMAR $)$ somewhat improved to $\underline{0.45 \pm 0.32}$ at three months $(P=0.156)$. However, the improvement in visual acuity then subsided, and returned to the pretreatment value at 12 months $\underline{(0.54 \pm 0.39)}$. Conclusions: In eyes with PCV, IVB can reduce the exudative change and can maintain visual function for at least one year.

Key words: age-related macular degeneration; bevacizumab; polypoidal choroidal vasculopathy 


\section{Introduction}

It is generally thought that vascular endothelial growth factor (VEGF) plays a central role in the formation and development of choroidal neovascularization (CNV) in age-related macular degeneration (AMD). ${ }^{1,2}$ Visual prognosis in eyes with subfoveal

5 CNV secondary to AMD is poor. Recently, a number of reports have shown promising effects of bevacizumab (Avastin; Genentech, South San Francisco, California), which is a recombinant humanized monoclonal antibody that binds to VEGF, for CNV secondary to AMD. ${ }^{3}$

Polypoidal choroidal vasculopathy (PCV) was first described as a new clinical entity with a unique form of choroidal vascular abnormality, which is characterized by a branching vascular network that terminates in polypoidal lesions seen on indocyanine green angiography. ${ }^{4-6}$ However, Maruko et al. ${ }^{7}$ recently reported that $5.5 \%$ of patients with exudative AMD showed PCV lesions in one eye and typical AMD lesions in the other eye. It is still controversial whether PCV is a distinct clinical entity or represents an unusual form of AMD. ${ }^{8} \quad$ Similar to $A M D,{ }^{9}$ it has been reported that VEGF concentrations in aqueous humor are markedly increased in eyes with PCV. ${ }^{10}$ The involvement of VEGF in the formation of PCV is also suggested by histologic specimens. ${ }^{11}$ Therefore, it may well be that anti-VEGF therapy is effective for the treatment of PCV. 
Recently, several investigators have reported the effects of intravitreal bevacizumab (IVB) for the treatment of exudative PCV. ${ }^{12-19}$ Even though the polypoidal lesions sometimes persist after IVB injection, retinal thickness is reported to be decreased shortly after the initiation of such treatment. ${ }^{13-15,17}$ In eyes with PCV,

5 however, the effects of IVB on visual acuity (VA) remain controversial. ${ }^{13-15,17}$ In addition, because these previous reports have only a short follow-up period, limited information is available on the long-term efficacy of IVB in eyes with PCV. ${ }^{13}$ Accordingly, the study described herein was designed to study for at least one year the effects of IVB administered for the treatment of PCV. 


\section{Material and Methods}

For this interventional case series, we studied retrospectively the medical records of 17 eyes (16 patients) with active PCV that were treated with IVB at Kyoto University Hospital from October 2006 to April 2008. All eyes had a minimum follow-up period

5 of 12 months after the initial treatment with IVB. The eligibility criteria were active PCV with a visual disturbance and treatment with IVB. The diagnosis of PCV was based on indocyanine green angiography, which shows a branching vascular network that terminates in polypoidal swelling. In the current study, $\underline{15}$ eyes showed either a polypoidal lesion, a branching vascular network, or type 2 CNV beneath the center of the fovea. Two eyes showed a serous pigment epithelial detachment (PED) just beneath the fovea with adjacent juxtafoveal polypoidal lesions. Eyes with other macular abnormalities (i.e., AMD, pathologic myopia, idiopathic CNV, presumed ocular histoplasmosis, angioid streaks and other secondary CNV) were excluded from the current study. In the present study, pseudophakic eyes were included, but eyes with a history of vitrectomy were excluded. Eyes that had undergone photodynamic therapy (PDT) for PCV within four months were also excluded from the current study. The current study was approved by the Institutional Review Board at Kyoto University Graduate School of Medicine and adhered to the tenets of the Declaration of Helsinki. Written informed consent was obtained from each patient. 
In the study described herein, patients who had a subjective visual disturbance due to subfoveal or juxtafoveal PCV were offered IVB, the dosage of which was 1.25 $\mathrm{mg} / 0.05 \mathrm{ml}$ per injection. All injections were performed in a sterile manner and prophylactic topical antibiotics were applied for 1 week after IVB injection. As initial

5 treatment, $\underline{10}$ eyes were treated with a single injection of bevacizumab and $\underline{7}$ eyes were treated with 3 monthly injections. Each patient was scheduled to visit our clinic for an examination at a 1-2 month interval. After the initial treatment, when recurrent or residual exudative changes due to PCV were seen by optical coherence tomography (OCT) examination or on a fundus examination, additional IVB injections were given.

At each visit, all patients underwent a comprehensive ophthalmologic examination, including best-corrected VA testing, intraocular pressure measurement, indirect ophthalmoscopy, slitlamp biomicroscopy with a contact lens, fundus photography, and OCT. Best-corrected VA was measured with a Landolt chart. Fluorescein and indocyanine green angiography were performed on each patient using a confocal laser scanning system (HRA-2; Heidelberg Engineering, Heidelberg, Germany) before and at 3-6 months after the treatment. Additional angiography was performed as necessary.

In this study, spectral-domain OCT was used at the baseline examination and at 
follow-up visits. At each visit, all patients underwent examination by OCT of cross-sections centered on the fovea as well as OCT of additional sections.

Because eyes with PCV often showed serosanguineous pigment epithelial detachment, foveal thickness was defined as the distance between the inner surface

5 of the neurosensory retina and the thin straight line of Bruch's membrane. ${ }^{14}$

All values are presented as mean \pm standard deviation. For statistical analysis, best-corrected VA measured with a Landolt chart was converted to a logarithm of the minimal angle of resolution (logMAR). Paired $t$-test was used to evaluate changes in VA and foveal thickness. In each eye, VA was considered to be improved or deteriorated when the change in VA (logMAR) was greater than 0.3. Statistical software (Statview 5.0; SAS Institute, Cary, North Carolina) was used for statistical analyses. A $P$ value $<0.05$ was considered to be statistically significant. 


\section{RESULTS}

In the current study, $\underline{17}$ eyes of $\underline{16}$ patients ( $\underline{14}$ men and $\underline{2}$ women), ranging in age from $\underline{60}$ to 85 years $(\underline{74.8 \pm 6.2}$ years $)$, underwent IVB injection for the treatment of subfoveal or juxtafoveal PCV (Table 1). While $\underline{3}$ of $\underline{17}$ eyes had undergone PDT

5 previously, all eyes showed active exudative changes due to their PCV. No eyes had been treated previously with either local or systemic anti-VEGF therapy.

Examination just before initiation of the IVB treatment showed polypoidal lesions in $\underline{14}$ eyes, branching vascular network in all $\underline{17}$ eyes, type $2 \mathrm{CNV}$ in 3 eyes, serous retinal detachment in $\underline{9}$ eyes, and PED in $\underline{10}$ eyes. Of the $\underline{17}$ eyes, subfoveal findings were polypoidal lesions in 4 eyes, a branching vascular network in 11 eyes, and PED in 2 eyes. Pretreatment VA on a Landolt chart ranged from 0.09 to 1.2 (median, 0.3 ): $\underline{\text { five }}$ of the $\underline{17}$ eyes had a VA of better than 0.5 (median, $\underline{0.8}$ ) before treatment. Fifteen eyes received additional IVB injections. Mean total injections was $\underline{4.0 \pm 1.7}$, ranging from 1 to 6 . Follow-up after the initiation of IVB ranged from 12 to 30 months (mean, $\underline{20.7 \pm 5.7}$ months).

Figure 1 shows the change in VA and in foveal thickness after IVB injection. IVB treatment reduced substantially the exudative change after initiation of the treatment in $\underline{13}$ eyes (Fig. 2). The mean foveal thickness $\underline{492 \pm 205} \mu \mathrm{m}$ before treatment) was decreased significantly to $\underline{384 \pm 181} \mu \mathrm{m}(P=\underline{0.0008})$ at three months. 
After this successful initial treatment, however, six eyes showed a recurrence of the exudative change (Fig. 3). Repeated angiography confirmed a recurrence of polypoidal lesions in two eyes. With additional IVB, foveal thickness at 12 months $\underline{(392 \pm 203 \mu \mathrm{m}) \text { was still reduced significantly compared with pretreatment }}$

$5 \quad$ measurements of thickness $(P=0.0270)$. The mean VA before treatment $(\underline{0.54 \pm}$ $\underline{0.38}$ in logMAR) also somewhat improved, being $\underline{0.45 \pm 0.32}(P=0.156)$ at three months; the difference was not statistically significant. Unfortunately, the improvement in VA subsided thereafter. At 12 months, mean VA had returned to the pretreatment value $(\underline{0.54 \pm 0.39})$. At 12 months, two eyes showed a significant improvement in VA and no eye showed a significant decrease in VA. Whether a single injection of IVB or 3 monthly IVB injections were given as the initial treatment, there was no significant difference in changes of either the VA or of the foveal thickness.

In the current study, $\underline{14}$ eyes showed polypoidal lesions just before treatment with IVB, while postoperative angiography showed the polypoidal lesions to have disappeared in $\underline{9}$ eyes (Fig. 4). In no eye, however, had the branching vascular network disappeared. In addition, 3 eyes showed type 2 CNV just before the initiation of IVB. On postoperative angiography, leakage from the type 2 CNV had disappeared completely in 2 eyes and was substantially decreased in the other eye. 
Figure 5 shows the change in VA and in foveal thickness after IVB in $\underline{5}$ eyes that had a good initial VA (better than 0.5 on a Landolt chart). The exudative change was reduced after the initiation of IVB treatment in all $\underline{5}$ of these eyes, and after this successful initial treatment, recurrent exudative change or macular edema was

5 treated with additional IVB injections. The mean VA $(\underline{0.10 \pm 0.11}$ in $\log M A R$ before treatment) was maintained at 12 months $(\underline{0.13 \pm 0.12})$ in all 5 eyes; one eye, however, developed a tear of the retinal pigment epithelium at 3 months after 3 monthly IVB injections (Fig. 6). 


\section{Discussion}

PCV is thought to have a better visual prognosis than does AMD. ${ }^{20}$ However, when the abnormal choroidal vasculature with exudative change extends to the subfoveal area, visual prognosis usually is poor. Direct laser photocoagulation has been

5 applied to eyes with PCV. ${ }^{21}$ However, unless the entire lesion including the polypoidal lesion and a branching vascular network is successfully coagulated, the polypoidal lesion and exudative change often recur, resulting in decreased VA. ${ }^{22}$ A number of studies, however, have shown encouraging results of PDT for PCV. ${ }^{23-25}$ In a report by Chan et al., ${ }^{23}$ PDT resulted in stable or improved VA in $95 \%$ of eyes with PCV one year after treatment. However, recent studies have shown that visual prognosis of PCV treated with PDT is not so promising. ${ }^{26-28}$ One or more years after successful treatment with PDT, some eyes have a recurrence of PCV and, consequently, a decrease in VA.

Similar to AMD, some reports have suggested that VEGF is involved in the

15 development of vascular lesions in eyes with PCV. ${ }^{10,11}$ Recently, several investigators have reported the short-term effects of anti-VEGF therapy for the treatment of exudative PCV. ${ }^{12-19,29}$ Originally, Ghajarnia et al. ${ }^{12}$ reported a case of refractory PCV that was successfully treated with a single injection of IVB. Most subsequent reports have shown that IVB reduces the exudative change resulting from 
PCV lesions shortly after the initiation of treatment, while exerting also an effect on the vascular lesions of PCV. ${ }^{13-15,17}$ However, its effect on VA remains controversial. ${ }^{13-15,}$

17 Gomi et al. ${ }^{14}$ reported no improvement in VA at three months after treatment of PCV with IVB. In contrast, Lai et al. ${ }^{13}$ and Song et al. ${ }^{15}$ reported a significant

5 improvement in VA at three months. However, because these reports each consisted of a small number of patients, selection of patients may have influenced the reported efficacy of treatment. ${ }^{13-15,17}$ In addition, because each of these reports had a relatively short follow-up, ${ }^{14,15,17}$ the long-term effect of IVB on eyes with PCV is uncertain. months (mean, $20.7 \pm 5.7$ months). Similar to the previous reports, IVB treatment reduced substantially at three months the exudative change, ${ }^{13,15,17}$ and with additional IVB to recurrent exudative change, the decreased foveal thickness could be maintained for 12 months. With IVB, mean VA was also somewhat improved at three months. This improvement in VA then subsided, and the mean VA returned to the pretreatment level at 12 months, even though the recurrent exudative change was often treated successfully with additional IVB. Judging from the current study, eyes with PCV that are treated with IVB can be expected to maintain the pretreatment VA for at least one year. 
After the initial IVB treatment, our patients received additional IVB when a recurrent or persistent exudative change was seen. In both the MARINA ${ }^{30}$ and $\mathrm{ANCHOR}^{31}$ studies, monthly treatments with ranibizumab successfully improved VA in eyes with AMD, while the PIER study ${ }^{32}$ showed that less frequent scheduled injections

5 did not maintain the improvement in VA that was made with the initial monthly injections. However, it is still controversial whether additional treatment with ranibizumab to recurrent or persistent exudative change does maintain the improvement in VA that had been achieved with the initial treatment. ${ }^{33}$ The PrONTO study ${ }^{33}$ showed a promising effect, although the SAILOR study did not. Even with additional IVB, our patients did not show at the final visit the VA improvement that they had gained at three months. Delayed treatment to the recurred exudative change may cause the damage of the photoreceptor cells, resulting in the poor visual outcome. It is possible that more frequent IVB injections might result in more promising final VA. In addition, while our patients were scheduled to visit our clinic for an examination at a 1-2 month interval, monthly examinations would be essential to improve visual prognosis.

In the current study, IVB did result in a regression of type 2 CNV secondary to PCV. In $\underline{36 \%}$ of eyes, however, the polypoidal lesions did not disappear with IVB, and it did not diminish at all the branching vascular network. The reason why IVB is 
less effective against the PCV lesion itself is uncertain. One possible explanation is the location of the vascular lesion of PCV, which is situated beneath the retinal pigment epithelium. $^{8} \quad$ Another reason may be that VEGF is more weakly involved in vascular components of PCV than it is in CNV secondary to AMD. Based on the

5 visual prognosis of eyes with PCV that received other treatments (i.e., laser photocoagulation or PDT), the recurrence of the vascular lesion may be the most crucial for the long-term treatment effect on $\mathrm{PCV} .^{22,26-28}$ In PCV, repeated treatment with IVB may be necessary to avoid recurrence. Furthermore, combination therapy with PDT might be superior to IVB monotherapy in order to reduce recurrences. ${ }^{16,18}$ The current study included five eyes with relatively good initial VA (better than 0.5 on a Landolt chart), but as all eyes showed an exudative change with subfoveal or juxtafoveal PCV, visual prognosis may be poor without any treatments. PDT may cause severe hemorrhagic complications in eyes with $\mathrm{PCV},{ }^{34}$ and, in the clinical setting, it may be difficult to treat with PDT those eyes that have PCV and good VA.

15 In eyes with PCV, IVB might be safer than is PDT. Previously, Gomi et al. ${ }^{14}$ used IVB in eyes with PCV that had good VA and maintained the initial VA for three months. In the current study in which IVB was used, all 5 eyes with good initial VA maintained their VA for 12 months. However, one of these five eyes developed a tear in the retinal pigment epithelium. Eyes with PCV often show a serosanguineous PED, ${ }^{35}$ 
and when a large PED is seen beneath the fovea, it should be kept in mind that development of a tear in the retinal pigment epithelium is a possible complication of IVB. ${ }^{36}$

Limitations of the current study are the various treatment regimens used and the

5 small sample size. However, despite these short-comings, our findings suggest that IVB can maintain VA for at least 12 months in eyes with PCV. In addition, Reche-Frutos et al. ${ }^{29}$ recently reported the promising short-term effects of ranibizumab for PCV, and anti-VEGF therapy remains an option for the treatment of PCV. Therefore, prospective studies with long-term follow-up are needed to evaluate the safety and efficacy of IVB for the treatment of PCV. 


\section{References}

1. Kvanta A, Algvere PV, Berglin L, Seregard S. Subfoveal fibrovascular membranes in age-related macular degeneration express vascular endothelial growth factor. Invest Ophthalmol Vis Sci 1996;37:1929-1934.

2. Ciulla TA, Rosenfeld PJ. Antivascular endothelial growth factor therapy for neovascular age-related macular degeneration. Curr Opin Ophthalmol $2009 ; 20: 158-165$.

3. Avery RL, Pieramici DJ, Rabena MD, Castellarin AA, Nasir MA, Giust MJ. Intravitreal bevacizumab (Avastin) for neovascular age-related macular degeneration. Ophthalmology 2006;113:363-372.

4. Kleiner RC, Brucker AJ, Johnston RL. The posterior uveal bleeding syndrome. Retina 1990;10:9-17.

5. Stern RM, Zakov ZN, Zegarra H, Gutman FA. Multiple recurrent serosanguineous retinal pigment epithelial detachments in black women. Am J Ophthalmol 1985;100:560-569.

6. Yannuzzi LA, Sorenson J, Spaide RF, Lipson B. Idiopathic polypoidal choroidal vasculopathy (IPCV). Retina 1990;10:1-8.

7. Maruko I, lida T, Saito M, Nagayama D, Saito K. Clinical characteristics of exudative age-related macular degeneration in Japanese patients. Am J 
Ophthalmol 2007;144:15-22.

8. Ciardella AP, Donsoff IM, Huang SJ, Costa DL, Yannuzzi LA. Polypoidal choroidal vasculopathy. Surv Ophthalmol 2004;49:25-37.

9. Wells JA, Murthy R, Chibber R, et al. Levels of vascular endothelial growth factor are elevated in the vitreous of patients with subretinal neovascularisation. $\mathrm{Br} \mathrm{J}$ Ophthalmol 1996;80:363-366.

10. Tong JP, Chan WM, Liu DT, et al. Aqueous humor levels of vascular endothelial growth factor and pigment epithelium-derived factor in polypoidal choroidal vasculopathy and choroidal neovascularization. Am J Ophthalmol 2006;141:456-462.

11. Matsuoka M, Ogata N, Otsuji T, Nishimura T, Takahashi K, Matsumura M. Expression of pigment epithelium derived factor and vascular endothelial growth factor in choroidal neovascular membranes and polypoidal choroidal vasculopathy. Br J Ophthalmol 2004;88:809-815.

12. Ghajarnia M, Kurup S, Eller A. The therapeutic effects of intravitreal bevacizumab in a patient with recalcitrant idiopathic polypoidal choroidal vasculopathy. Semin Ophthalmol 2007;22:127-131.

13. Lai TY, Chan WM, Liu DT, Luk FO, Lam DS. Intravitreal bevacizumab (Avastin) with or without photodynamic therapy for the treatment of polypoidal choroidal 
vasculopathy. Br J Ophthalmol 2008;92:661-666.

14. Gomi F, Sawa M, Sakaguchi H, et al. Efficacy of intravitreal bevacizumab for polypoidal choroidal vasculopathy. Br J Ophthalmol 2008;92:70-73.

15. Song JH, Byeon SH, Lee SC, Koh HJ, Kwon OW. Short-term safety and efficacy of a single intravitreal bevacizumab injection for the management of polypoidal choroidal vasculopathy. Ophthalmologica 2009;223:85-92.

16. Pai SA, Shetty R. Sequential therapy with intravitreal bevacizumab and photodynamic therapy for idiopathic polypoidal choroidal vasculopathy. Acta Ophthalmol, in press.

17. Lee SY, Kim JG, Joe SG, Chung H, Yoon YH. The therapeutic effects of bevacizumab in patients with polypoidal choroidal vasculopathy. Korean $\mathrm{J}$ Ophthalmol 2008;22:92-99.

18. Zuo C, Wen F, Li J, Liu Y, Li M. Transitions of multifocal electroretinography following combined intravitreal bevacizumab and photodynamic therapy for polypoidal choroidal vasculopathy. Doc Ophthalmol 2009;119:29-36.

19. Cho M, Barbazetto IA, Freund KB. Refractory neovascular age-related macular degeneration secondary to polypoidal choroidal vasculopathy. Am J Ophthalmol 2009;148:70-78.

20. Sho K, Takahashi K, Yamada H, et al. Polypoidal choroidal vasculopathy: 
incidence, demographic features, and clinical characteristics. Arch Ophthalmol 2003;121:1392-1396.

21. Nishijima K, Takahashi M, Akita J, et al. Laser photocoagulation of indocyanine green angiographically identified feeder vessels to idiopathic polypoidal choroidal vasculopathy. Am J Ophthalmol 2004;137:770-773.

22. Yuzawa M, Mori R, Haruyama M. A study of laser photocoagulation for polypoidal choroidal vasculopathy. Jpn J Ophthalmol 2003;47:379-384.

23. Chan WM, Lam DS, Lai TY, et al. Photodynamic therapy with verteporfin for symptomatic polypoidal choroidal vasculopathy: one-year results of a prospective case series. Ophthalmology 2004;111:1576-1584.

24. Otani A, Sasahara M, Yodoi Y, et al. Indocyanine green angiography: guided photodynamic therapy for polypoidal choroidal vasculopathy. Am J Ophthalmol 2007;144:7-14.

25. Gomi F, Ohji M, Sayanagi K, et al. One-year outcomes of photodynamic therapy in age-related macular degeneration and polypoidal choroidal vasculopathy in Japanese patients. Ophthalmology 2008;115:141-146.

26. Kurashige Y, Otani A, Sasahara M, et al. Two-year results of photodynamic therapy for polypoidal choroidal vasculopathy. Am J Ophthalmol 2008;146:513-519. 
27. Akaza E, Mori R, Yuzawa M. Long-term results of photodynamic therapy of polypoidal choroidal vasculopathy. Retina 2008;28:717-722.

28. Wakabayashi T, Gomi F, Sawa M, Tsujikawa M, Tano Y. Marked vascular changes of polypoidal choroidal vasculopathy after photodynamic therapy. Br J Ophthalmol 2008;92:936-940.

29. Reche-Frutos J, Calvo-Gonzalez C, Donate-Lopez J, Garcia-Feijoo J, Leila M, Garcia-Sanchez J. Short-term anatomic effect of ranibizumab for polypoidal choroidal vasculopathy. Eur J Ophthalmol 2008;18:645-648.

30. Rosenfeld PJ, Brown DM, Heier JS, et al. Ranibizumab for neovascular age-related macular degeneration. N Engl J Med 2006;355:1419-1431 .

31. Brown DM, Kaiser PK, Michels M, et al. Ranibizumab versus verteporfin for neovascular age-related macular degeneration. $\mathrm{N}$ Engl J Med $2006 ; 355: 1432-1444$.

32. Regillo CD, Brown DM, Abraham P, et al. Randomized, double-masked, sham-controlled trial of ranibizumab for neovascular age-related macular degeneration: PIER Study year 1. Am J Ophthalmol 2008;145:239-248.

33. Lalwani GA, Rosenfeld PJ, Fung AE, et al. A variable-dosing regimen with intravitreal ranibizumab for neovascular age-related macular degeneration: year 2 of the PrONTO study. Am J Ophthalmol 2009;148:43-58. 
34. Hirami Y, Tsujikawa A, Otani A, et al. Hemorrhagic complications after photodynamic therapy for polypoidal choroidal vasculopathy. Retina $2007 ; 27: 335-341$

35. Tsujikawa A, Sasahara M, Otani A, et al. Pigment epithelial detachment in polypoidal choroidal vasculopathy. Am J Ophthalmol 2007;143:102-111.

36. Peng $\mathrm{CH}$, Cheng $\mathrm{CK}$, Chiou $\mathrm{SH}$. Retinal pigment epithelium tear after intravitreal bevacizumab injection for polypoidal choroidal vasculopathy. Eye 2009;23:2126-2129. 


\section{Figure legend}

Figure 1. Mean foveal thickness (A) and mean visual acuity (VA) in logMAR fashion (B) in eyes with polypoidal choroidal vasculopathy treated with intravitreal injections of bevacizumab. ${ }^{*} P<0.01,{ }^{\dagger} P<0.05$, compared with pretreatment values. $\quad$ Mo $=$ 5 month(s).

Figure 2. Polypoidal choroidal vasculopathy that was treated successfully with intravitreal injections of bevacizumab (patient 2). (A) Funduscopic photograph shows massive subfoveal exudation with surrounding subretinal hemorrhage. Visual acuity of the left eye was 0.15 .

(B) Horizontal (upper) and vertical (lower) optical coherence tomographic images along white arrows shown in the fundus photograph reveal a sharp protrusion of retinal pigment epithelium with overlying subretinal exudate. (C) Fluorescein angiography reveals classic subfoveal choroidal neovascularization (CNV). (D) Indocyanine green angiography reveals a branching vascular network terminating in two polypoidal lesions (arrow) and type 2 CNV (long arrow). This eye was treated with 3 monthly injections of bevacizumab and 3 additional injections. (E) Twenty-four months after the initial treatment, funduscopic photograph shows fibrosis and surrounding atrophy of retinal pigment epithelium. Visual acuity remained at 0.15 . (F) Horizontal (upper) and vertical (lower) optical 
coherence tomographic images along white arrows shown in the fundus photograph

reveal minimal exudative change with a protrusion of retinal pigment epithelium.

Fluorescein angiography shows minimal leakage. (H) Indocyanine green

angiography reveals disappearance of both the polypoidal lesions and the type $2 \mathrm{CNV}$,

5 but the branching vascular network is still visible.

Figure 3. Recurrence of exudative change after successful treatment of the polypoidal lesion with intravitreal injections of bevacizumab (patient $\underline{15}$ ).

Funduscopic photograph shows large pigment epithelial detachment with reddish

(C) Indocyanine green angiography shows a branching vascular network terminating in many polypoidal lesions. (D) Horizontal (left) and vertical (right) optical coherence tomographic images along white arrows shown in the fundus photograph reveal a sharp protrusion of the retinal pigment epithelium with overlying subretinal exudates and retinal edema. This eye was treated with 3 monthly injections of bevacizumab.

(E) Four months after the initial treatment, funduscopic examination shows a decrease in the exudative change. Visual acuity had improved to 0.8. (F) Fluorescein angiography reveals no classic CNV. (G) Indocyanine green angiography reveals disappearance of the 
polypoidal lesions, but the branching vascular network is still visible. $(\mathbf{H})$ Horizontal (left) and vertical (right) optical coherence tomographic images along white arrows indicated in the fundus photograph show substantially reduced exudative change.

Horizontal (left) and vertical (right) optical coherence tomographic images obtained at

512 months show a recurrence of the subretinal fluid. Visual acuity at this time was 0.7 .

Figure 4. Intravitreal injections of bevacizumab improved visual acuity in spite of no change in the polypoidal lesions or in the branching vascular network (patient $\underline{9}$ ).

Fluorescein angiography shows classic choroidal neovascularization (CNV).

Indocyanine green angiography reveals a branching vascular network that terminates in two polypoidal lesions (arrows). (C) Horizontal (left) and vertical (right) optical coherence tomographic images along white arrows shown in the fluorescein angiogram reveal a sharp protrusion of the retinal pigment epithelium with an overlying subretinal exudate. Visual acuity of this eye was 0.3 , and it was treated with 4 injections of bevacizumab. (D) Eight months after the initial treatment, fluorescein angiography shows minimal leakage. (E) Indocyanine green angiography shows unchanged polypoidal lesions and unchanged branching vascular network. (F) Horizontal (left) and vertical (right) optical coherence tomographic 
images show no exudative change but remaining cystoid spaces. Visual acuity of the eye at this time was 0.5. (G) Horizontal (left) and vertical (right) optical coherence tomographic images obtained at 19 months show no cystoid spaces.

Visual acuity was improved to 0.7 .

Figure 5. Mean foveal thickness (A) and mean visual acuity in logMAR fashion (B) in eyes with polypoidal choroidal vasculopathy treated with intravitreal injections of bevacizumab. All of these eyes had good pretreatment visual acuity (VA) (better than 0.5$) . \quad{ }^{*} P<0.05$, compared with pretreatment values. $\quad M o=$ month(s).

Figure 6. A tear of the retinal pigment epithelium (RPE) seen in polypoidal choroidal vasculopathy after intravitreal injections of bevacizumab (patient 16). (A) Funduscopic photograph shows a pigment epithelial detachment (PED) with a large reddish orange nodule. Visual acuity of this eye was 1.2. (B) Fluorescein angiography shows hyperfluorescence reflecting the PED. (C) Indocyanine green angiography reveals a branching vascular network terminating in two polypoidal lesions (arrows) at the edge of the PED. (D) Horizontal (left) and vertical (right) optical coherence tomographic images along white arrows in the fundus photograph show a sharp protrusion of the retinal pigment epithelium due to a polypoidal lesion 
(arrow) at the edge of the PED. The eye was treated with 3 monthly injections of bevacizumab. (E) Three months after the initial treatment, funduscopic examination shows a large tear of the RPE (arrowheads). Visual acuity had decreased to 0.8 . (F) Fluorescein angiography shows a window defect due to a tear of the RPE.

$5 \quad$ No polypoidal lesions are seen on indocyanine green angiography. Folded RPE shows a blockage of the fluorescence (arrow). (H) Horizontal (left) and vertical (right) optical coherence tomographic images along white arrows shown in the fundus photograph reveal increased choroidal fluorescence due to a lack of RPE (arrowheads). At 12 months, visual acuity had decreased to 0.4 . 
Table 1. Characteristics of eyes with polypoidal choroidal vasculopathy treated with IVB

\begin{tabular}{|c|c|c|c|c|c|c|c|c|c|c|c|c|}
\hline \multirow[b]{2}{*}{ Patient } & \multirow{2}{*}{$\begin{array}{c}\text { Age / } \\
\text { Gender/ } \\
\text { RL }\end{array}$} & \multirow[b]{2}{*}{$\begin{array}{l}\text { Previous } \\
\text { treatment }\end{array}$} & \multicolumn{3}{|c|}{ Pretreatment condition } & \multirow[b]{2}{*}{ Initial IVB } & \multirow{2}{*}{$\begin{array}{c}\text { Total } \\
\text { number } \\
\text { of IVB }\end{array}$} & \multicolumn{3}{|c|}{ Final condition } & \multirow[b]{2}{*}{$\begin{array}{l}\text { Follow-up } \\
\text { (Mos) }\end{array}$} & \multirow[b]{2}{*}{$\begin{array}{l}\text { Compli- } \\
\text { cations }\end{array}$} \\
\hline & & & $\begin{array}{l}\text { Subfoval } \\
\text { lesion }\end{array}$ & VA & $\begin{array}{c}\text { Foveal } \\
\text { thickness }(\mu \mathrm{m})\end{array}$ & & & VA & $\begin{array}{c}\text { Foveal } \\
\text { thickness }(\mu \mathrm{m})\end{array}$ & Retinal condition & & \\
\hline 1 & $75 / \mathrm{M} / \mathrm{R}$ & None & BVN & 0.3 & 184 & 3 monthly injections & 4 & 0.4 & 140 & Dry & 30 & None \\
\hline 2 & 68/F/L & None & PL & 0.15 & 850 & 3 monthly injections & 6 & 0.15 & 349 & Fibrosis & 24 & None \\
\hline \multirow[t]{2}{*}{3} & $80 / \mathrm{M} / \mathrm{R}$ & None & BVN & 0.1 & 358 & Single injection & 5 & 0.1 & 763 & Exudative & 27 & None \\
\hline & $80 / \mathrm{M} / \mathrm{L}$ & None & BVN & 0.5 & 450 & Single injection & 5 & 0.2 & 756 & Exudative & 23 & None \\
\hline 4 & $74 / \mathrm{M} / \mathrm{R}$ & PDT & BVN & 0.1 & 415 & Single injection & 3 & 0.1 & 276 & Slightly exudative & 26 & None \\
\hline 5 & 79/M/L & PDT & BVN & 0.09 & 500 & Single injection & 2 & 0.06 & 204 & Fibrosis & 26 & None \\
\hline 6 & $78 / F / R$ & None & BVN & 0.1 & 250 & Single injection & 2 & 0.2 & 59 & Dry & 23 & None \\
\hline 7 & $82 / \mathrm{M} / \mathrm{R}$ & None & PL & 0.5 & 666 & Single injection & 3 & 0.3 & 280 & Dry & 22 & None \\
\hline 8 & $80 / \mathrm{M} / \mathrm{L}$ & None & $\mathrm{PL}$ & 0.3 & 363 & 3 monthly injections & 3 & 0.2 & 503 & Fibrosis & 20 & None \\
\hline 9 & $72 / \mathrm{M} / \mathrm{R}$ & None & BVN & 0.3 & 456 & Single injection & 6 & 0.7 & 232 & Dry & 19 & None \\
\hline 10 & $75 / \mathrm{M} / \mathrm{R}$ & None & BVN & 0.1 & 656 & Single injection & 4 & 0.06 & 652 & Exudative & 16 & None \\
\hline 11 & $85 / \mathrm{M} / \mathrm{R}$ & PDT x 2 & BVN & 0.2 & 969 & 3 monthly injections & 6 & 0.15 & 704 & Exudative & 13 & None \\
\hline 12 & $73 / \mathrm{M} / \mathrm{R}$ & None & PED & 0.7 & 345 & Single injection & 1 & 1.2 & 286 & Dry & 26 & None \\
\hline 13 & 60/M/L & None & PL & 0.8 & 633 & Single injection & 2 & 0.7 & 463 & Dry & 19 & None \\
\hline 14 & 73/M/R & None & BVN & 0.6 & 356 & 3 monthly injections & 6 & 0.7 & 322 & Slightly exudative & 13 & None \\
\hline 15 & $68 / \mathrm{M} / \mathrm{L}$ & None & BVN & 0.8 & 432 & 3 monthly injections & 6 & 0.7 & 381 & Dry & 13 & None \\
\hline 16 & 76/M/L & None & PED & 1.2 & 484 & 3 monthly injections & 4 & 0.4 & 330 & Dry & 12 & RPE tear \\
\hline
\end{tabular}

IVB, intravitreal injection of bevacizumab; PDT, photodynamic therapy; BVN, branching vascular network; PL, polypoidal lesion; PED, pigment epithelial detachment; RPE, retinal pigment epithelium; Mos, months. 

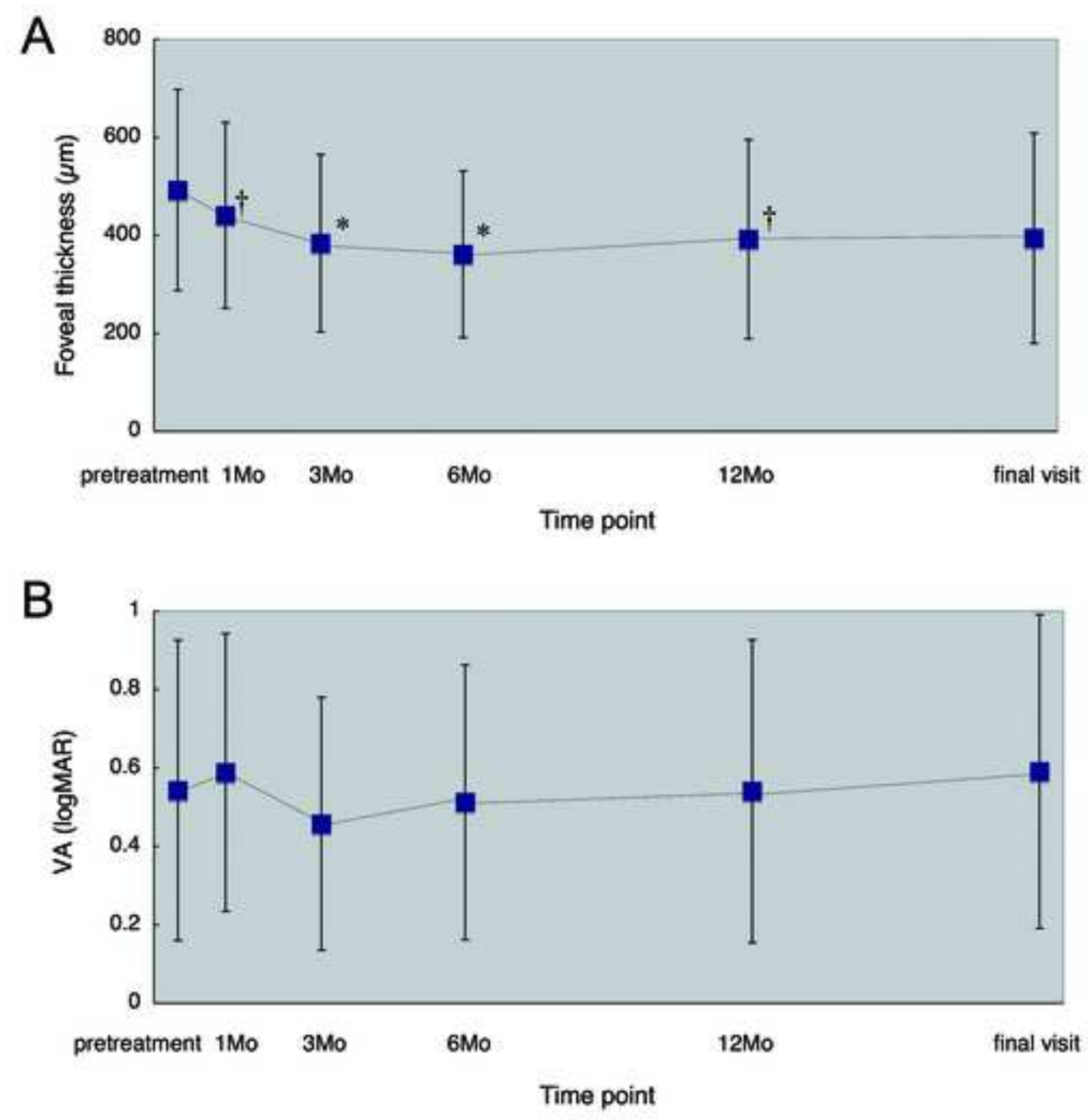


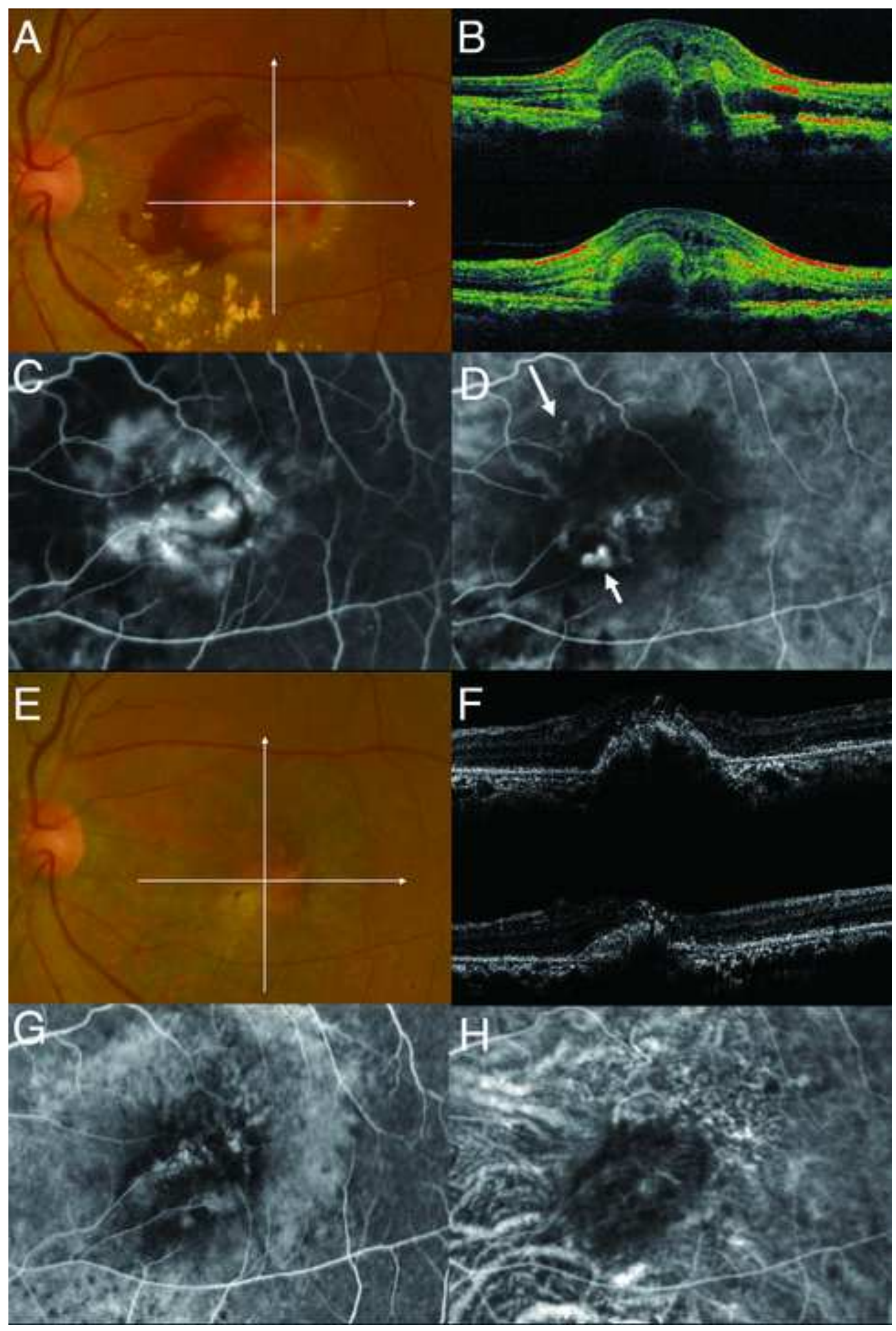




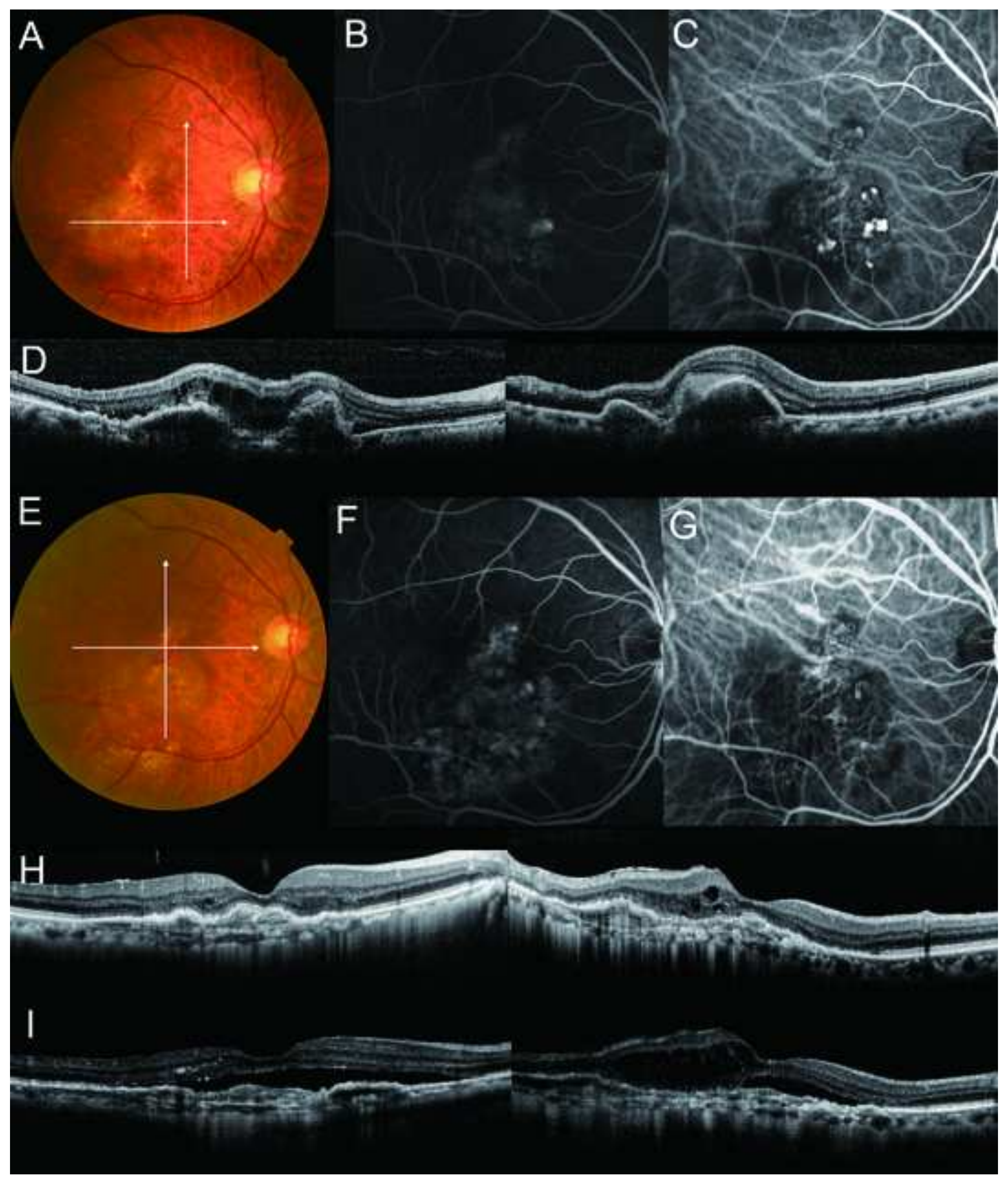



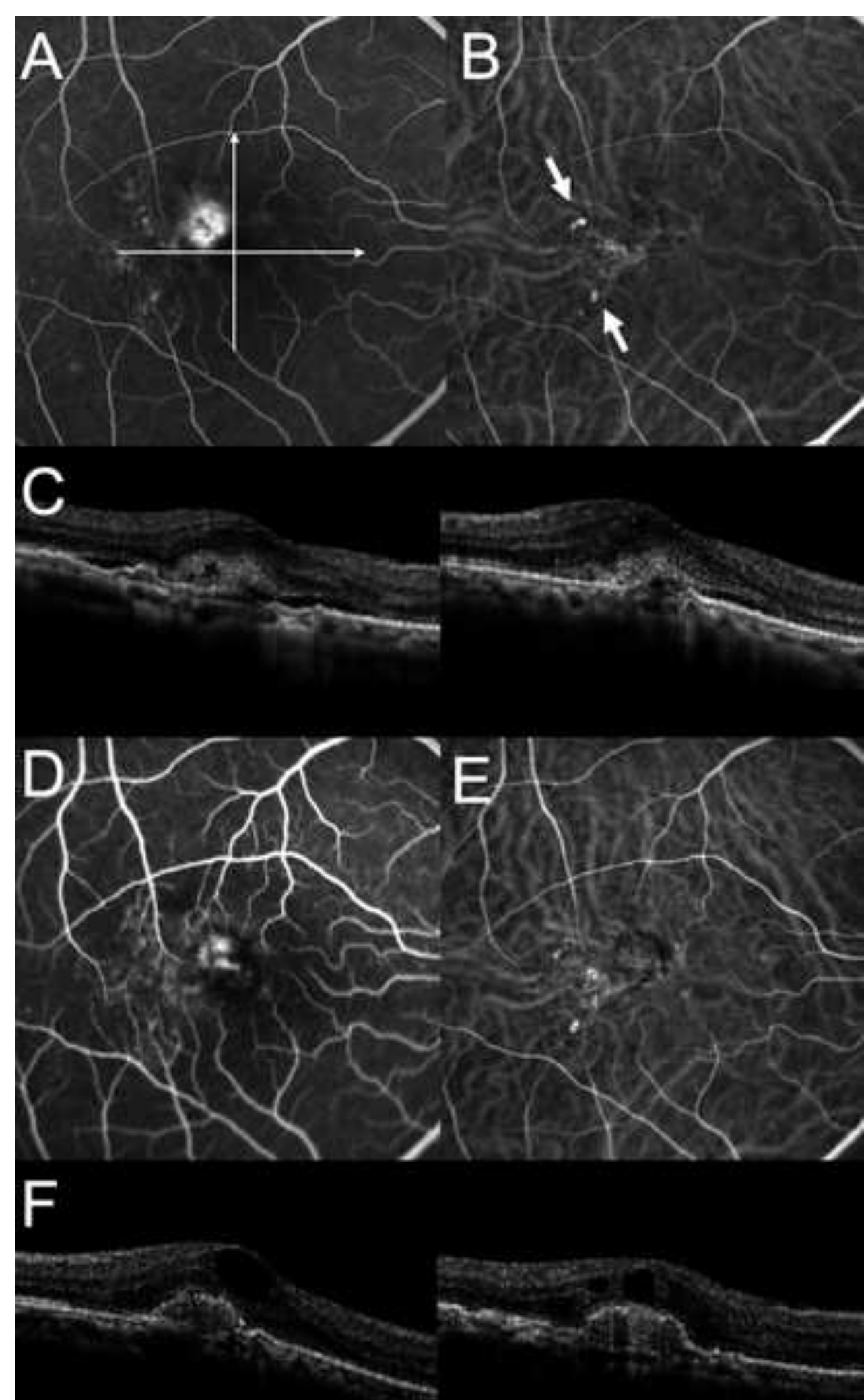

$G \quad$

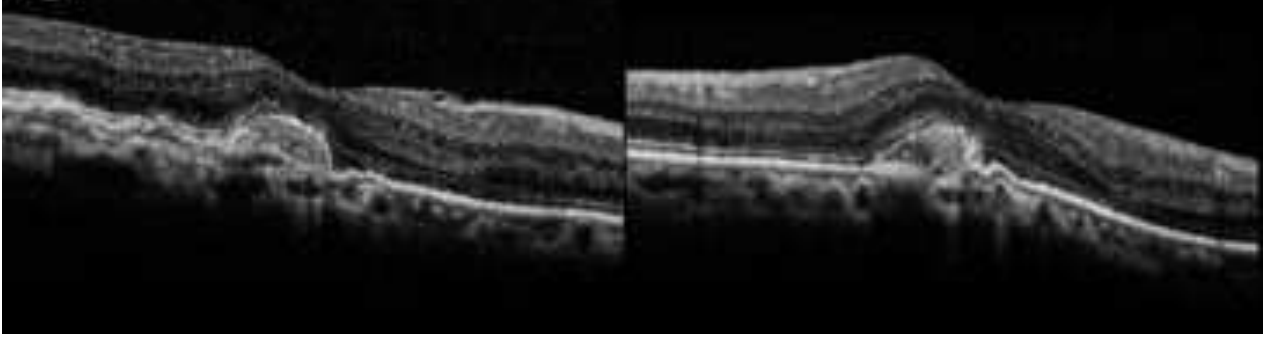



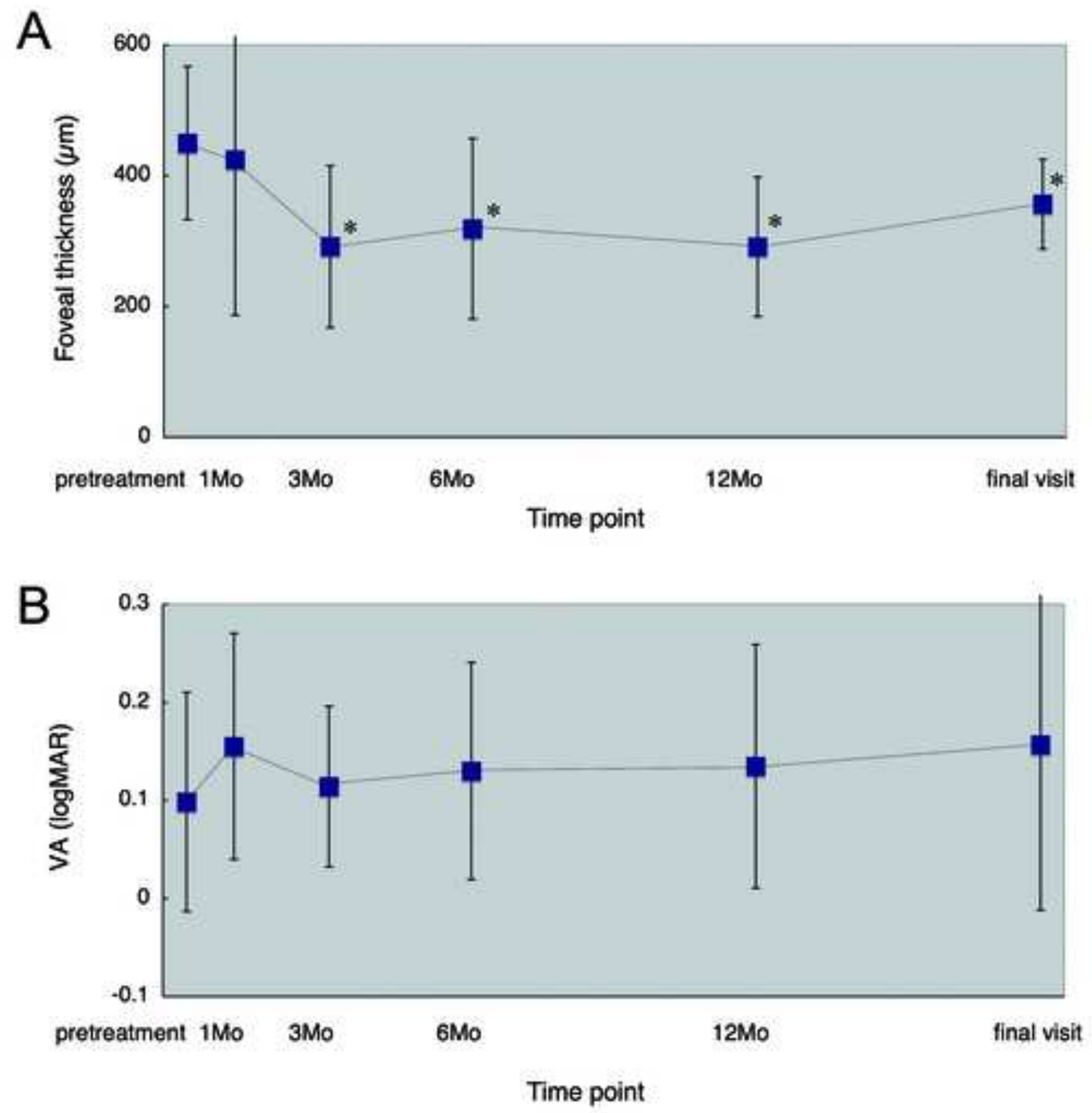